\title{
Linear Relative $n$-Widths for Linear Operators Preserving an Intersection of Cones
}

\author{
S. P. Sidorov \\ Department of Mechanics and Mathematics, Saratov State University, 410012 Saratov, Russia \\ Correspondence should be addressed to S. P. Sidorov; sidorovsp@yahoo.com
}

Received 17 January 2014; Accepted 4 April 2014; Published 29 May 2014

Academic Editor: Shyam L. Kalla

Copyright (C) 2014 S. P. Sidorov. This is an open access article distributed under the Creative Commons Attribution License, which permits unrestricted use, distribution, and reproduction in any medium, provided the original work is properly cited.

We introduce the definition of linear relative $n$-width and find estimates of linear relative $n$-widths for linear operators preserving the intersection of cones of $p$-monotonicity functions.

\section{Introduction}

In various applications of CAGD (computer-aided geometric design) it is necessary to approximate functions preserving its properties such as monotonicity, convexity, and concavity. The survey of the theory of shape-preserving approximation can be found in [1].

Let $X$ be a normed linear space and let $V$ be a cone in $X$ (a convex set, closed under nonnegative scalar multiplication). It is said that $f \in X$ has the shape in the sense of $V$ whenever $f \in V$. Let $X_{n}$ be a $n$-dimensional subspace of $X$. Classical problems of approximation theory are of interest in the theory of shape-preserving approximation as well:

(1) problems of existence, uniqueness, and characterization of the best shape-preserving approximation $g^{*} \epsilon$ $X_{n} \cap V$ of $f \in V$ defined by

$$
\left\|f-g^{*}\right\|_{X}=\inf _{g \in X_{n} \cap V}\|f-g\|_{X}
$$

(2) estimation of the deviation of $A \cap V$ from $X_{n} \cap V$, that is,

$$
E\left(A \cap V ; X_{n} \cap V\right)_{X}:=\sup _{f \in A \cap V} \inf _{g \in X_{n} \cap V}\|f-g\|_{X} ;
$$

(3) estimation of relative $n$-width of $A$ in $X$ with the constraint $V$

$$
d_{n}(A \cap V, V)_{X}:=\inf _{X_{n}} E\left(A \cap V ; X_{n} \cap V\right)_{X},
$$

the leftmost infimum taken over all affine subsets $X_{n}$ of dimension $\leq n$, such that $X_{n} \cap V \neq \varnothing$;

(4) estimation of linear relative $n$-widths with the constraint $V$ in $X$.

The notion of relative $n$-width (3) was first introduced in 1984 by Konovalov [2]. Though he considered a problem not connected with preserving shapes, the concept of relative $n$ width arises in the theory of shape-preserving approximation naturally. Of course, it is impossible to obtain $d_{n}(A \cap V, V)_{X}$ and determine optimal subspaces $X_{n}$ (if they exist) for all $A, V, X$. Nevertheless, some estimates of relative shapepreserving $n$-widths have been obtained in papers [3-5]. Estimates of relative (not necessary shape-preserving) widths have been obtained in works [6-11].

Let $A$ be a subset of $X$ and let $L: X \longrightarrow X$ be a linear operator. The value

$$
e(A, L):=\sup _{f \in A}\|f-L f\|_{X}=\sup _{f \in A}\|(I-L) f\|_{X}
$$

is the error of approximation of the identity operator $I$ by the operator $L$ on the set $A$.

Let $V$ be a cone in $X, V \neq \varnothing$. We will say that the operator $L$ preserves the shape in the sense of $V$, if $L(V) \subset V$. One might consider the problem of finding (if exists) a linear operator of finite rank $n$, which gives the minimal error of approximation of identity operator on some set over all finite rank $n$ linear operators $L$ preserving the shape in the sense $V$. It leads us naturally to the notion of linear relative $n$-width. 
In this paper we introduce the definition of linear relative $n$-width and find estimates of linear relative $n$-widths for linear operators preserving an intersection of cones of $p$ monotonicity functions.

\section{Notations and Definitions}

Let $B[0,1]$ denote the space of all real-valued bounded function, defined on $[0,1]$, with the uniform norm on $[0,1]$, and $\|f\|_{B[0,1]}=\sup _{x \in[0,1]}|f(x)|$. Denote by $B^{k}[0,1], k \geqslant 0$, the space of all real-valued functions, whose $k$ th derivative is bounded on $[0,1]$, endowed with the sup-norm

$$
\|f\|_{B^{k}[0,1]}=\sum_{0 \leq i \leq k} \frac{1}{i !} \sup _{x \in[0,1]}\left|D^{i} f(x)\right|
$$

where $D^{i}$ denotes the $i$ th differential operator, $D^{i} f(x)=$ $d^{i} f(x) / d x^{i}$, and $D^{0}=I$ is the identity operator, and the derivatives are taken from the right at 0 and from the left at 1 .

Denote by $C^{k}[0,1], k \geq 0$, the space of all real-valued and $k$-times continuously differentiable functions defined on $[0,1]$ equipped with sup-norm (5).

A continuous function $f:[0,1] \longrightarrow \mathbb{R}$ is said to be $k$ monotone, $k \geq 1$, on $[0,1]$ if and only if for all choices of $k+1$ distinct $t_{0}, \ldots, t_{k}$ in $[0,1]$ the inequality $\left[t_{0}, \ldots, t_{k}\right] f \geq 0$ holds, where $\left[t_{0}, \ldots, t_{k}\right] f$ denotes the $k$ th divided difference of $f$ at $0 \leq t_{0}<t_{1}<\cdots<t_{k} \leq 1$. Note that 2-monotone functions are just convex functions.

Let $\Delta^{p}$ denote the set of all $p$-monotone functions defined on $[0,1]$ and $\Delta^{0}:=\{f \in C[0,1]: f(t) \geq 0, t \in[0,1]\}$ denotes the cone of all nonnegative functions. If $f \in C^{p}[0,1]$, then $f \in \Delta^{p}$ if and only if $f^{(p)}(t) \geq 0, t \in[0,1]$. It is said that a linear operator $L$ of $C[0,1]$ into $C[0,1]$ preserves $p$ monotonicity, if $L\left(\Delta^{p}\right) \subset \Delta^{p}$.

Let $\sigma=\left(\sigma_{i}\right)_{i \geqslant 0}$ be a sequence of real numbers, $\sigma_{i} \epsilon$ $\{-1,0,1\}$, and let $h, k$ be two integers such that $0 \leqslant h \leqslant k$ and $\sigma_{h} \cdot \sigma_{k} \neq 0$. Denote

$$
\Delta^{h, k}(\sigma):=\left\{f \in C[0,1]: \sigma_{p} f \in \Delta^{p}, h \leq p \leq k\right\} .
$$

The cone $\Delta^{h, k}(\sigma)$ is the intersection of cones of $p$ monotonicity functions with $h \leq p \leq k$ taken with signs $\sigma_{p}$; that is, $\Delta^{h, k}(\sigma)=\cap_{p=h}^{k} \sigma_{p} \Delta^{p}$.

Denote $e_{i}(x)=x^{i}, i=0,1, \ldots$ Denote $\Pi_{m}:=$ $\operatorname{span}\left\{e_{0}, e_{1}, \ldots, e_{m}\right\}$ and $P_{m}:=\left\{f \in \Pi_{m}:\|f\|_{B^{m}[0,1]} \leq 1\right\}$.

Recall that a linear operator mapping $C[0,1]$ into a linear space of finite dimension $n$ is called an operator of finite rank $n$.

\section{The Example of Linear Operator Preserving the Cone $\Delta^{h, k}(\sigma)$}

This section gives an example of linear operator of finite rank $n$ preserving the cone $\Delta^{h, k}(\sigma)$ in the case $\sigma_{k}=\sigma_{k-2}=1$.
Denote $s(i):=\min \left\{s>i: \sigma_{s} \neq 0\right\}$ for $i \in\{h, \ldots, k-1\}$. Denote $t_{j}=j /(n-1), j=0,1, \ldots, n-1$. Let $\left(\delta_{p}\right)_{p=0}^{k-2}$ be the binary sequence defined by

$$
\delta_{p}= \begin{cases}0, & \text { if } p=k-2 \text { or if } \sigma_{p} \sigma_{s(p)} \neq-1, p \leq k-3 \\ 1, & \text { if } \sigma_{p} \sigma_{s(p)}=-1, p \leq k-3\end{cases}
$$

Let $k, n \in \mathbb{N}, n \geq k+2$, and $\Lambda_{n}^{h, k}: C^{k-2}[0,1] \longrightarrow C^{k-2}[0,1]$ be the linear operator defined by

$$
\Lambda_{n}^{h, k} f(x)=\left\{\begin{aligned}
\sum_{l=0}^{k-2} \frac{1}{l !} e_{l}(x) \\
\times\left(D^{l} f\left(t_{0}\right)\right. \\
\left.\quad-\delta_{l} \int_{t_{0}}^{t_{1}} D^{l+1}\left(\Lambda_{n}^{h, k} f-f\right)(t) d t\right) \\
+\frac{(n-1) e_{k-1}(x)}{(k-1) !} \\
\times\left[D^{k-2} f\left(t_{1}\right)-D^{k-2} f\left(t_{0}\right)\right] \\
\quad \text { if } x \in\left[t_{0}, t_{1}\right], \\
\sum \frac{1}{l !}\left(x-t_{j}\right)^{l} \\
\times\left(D^{l} \Lambda_{n}^{h, k} f\left(t_{j}\right)\right. \\
\left.\left.\quad-\delta_{l}\right]_{t_{j}}^{t_{j+1}} D^{l+1}\left(\Lambda_{n}^{h, k} f-f\right)(t) d t\right) \\
\quad(n-1) e_{k-1}\left(x-t_{j}\right) \\
\times\left[\begin{array}{l}
(k-1) ! \\
\times
\end{array}\right. \\
\quad \text { if } x \in\left(t_{j}, t_{j+1}\right], j=1,2, \ldots, n-2 .
\end{aligned}\right.
$$

Theorem 1. Consider that $\Lambda_{n}^{h, k}: C^{k-2}[0,1] \longrightarrow C^{k-2}[0,1]$ is a continuous linear operator of finite rank $n$, such that $\Lambda_{n}^{h, k}\left(\Delta^{h, k}(\sigma)\right) \subset \Delta^{h, k}(\sigma)$ and for all $0 \leq i \leq k-2$ there exists $c>0$ not depending on $n$ such that

$$
\sup _{f \in P_{k}}\left\|D^{i}\left(\Lambda_{n}^{h, k} f\right)-D^{i} f\right\|_{B[0,1]} \leq c n^{-2}
$$

Proof. Since $D^{k-2} \Lambda_{n}^{h, k} f$ is a piecewise linear function on $[0,1]$ with the set of breakpoints $\left\{\left(t_{j}, D^{k-2} f\left(t_{j}\right)\right)\right\}_{j=0, \ldots, n-1}$, then for every $f$ such that $D^{k-2} f \geq 0$ the inequality $D^{k-2} \Lambda_{n}^{h, k} f \geq 0$ holds. Moreover, $\Lambda_{n}^{h, k}\left(\sigma_{p} \Delta^{p}\right) \subset \sigma_{p} \Delta^{p}$ for $p=k+1, k+2$.

Note that for every $f \in \Delta^{k}$ we have $D^{k-2}\left(\Lambda_{n}^{h, k} f-f\right) \geq 0$ (since $D^{k-2} f$ is convex and $D^{k-2} \Lambda_{n}^{h, k} f$ is a piecewise linear interpolation). 
Let $f \in \Delta^{h, k}(\sigma)$ and suppose (by induction) that for $i \geq$ $h+1$ the inequality

$$
\sigma_{l} D^{l}\left(\Lambda_{n}^{h, k} f-f\right) \geq 0, \quad l=i+1, \ldots, k-2,
$$

holds on $\left[t_{0}, t_{1}\right]$. For any $x \in\left[t_{0}, t_{1}\right]$ we have

$$
\begin{aligned}
D^{i}\left(\Lambda_{n}^{h, k} f-f\right)(x)= & D^{i}\left(\Lambda_{n}^{h, k} f-f\right)\left(t_{0}\right) \\
& +\int_{t_{0}}^{x} D^{i+1}\left(\Lambda_{n}^{h, k} f-f\right)(z) d z .
\end{aligned}
$$

Consider the following three cases.

(1) If $\sigma_{i}=0$, then $\delta_{i}=0$ and it follows from $D^{i} \Lambda_{n}^{h, k} f(0)=$ $D^{i} f(0)$ that

$$
\operatorname{sgn} D^{i}\left(\Lambda_{n}^{h, k} f-f\right)=\operatorname{sgn} D^{i+1}\left(\Lambda_{n}^{h, k} f-f\right) .
$$

(2) If $\sigma_{i} \neq 0$ and $\sigma_{i}=\sigma_{s(i)}$, then it follows from (11) that

$$
\begin{aligned}
\operatorname{sgn} D^{i}\left(\Lambda_{n}^{h, k} f-f\right) & =\operatorname{sgn} D^{i+1}\left(\Lambda_{n}^{h, k} f-f\right)=\cdots \\
& =\operatorname{sgn} D^{s(i)}\left(\Lambda_{n}^{h, k} f-f\right)=\sigma_{i} .
\end{aligned}
$$
and

(3) If $\sigma_{i} \neq 0$ and $\sigma_{i}=-\sigma_{s(i)}$ then sgn $D^{i+1}\left(\Lambda_{n}^{h, k} f-f\right)=\sigma_{s(i)}$

$$
\begin{aligned}
D^{i}\left(\Lambda_{n}^{h, k} f-f\right)(x)= & D^{i}\left(\Lambda_{n}^{h, k} f-f\right)\left(t_{0}\right) \\
& +\int_{t_{0}}^{x} D^{i+1}\left(\Lambda_{n}^{h, k} f-f\right)(z) d z \\
= & -\int_{t_{0}}^{t_{1}} D^{i+1}\left(\Lambda_{n}^{h, k} f-f\right)(z) d z \\
& +\int_{t_{0}}^{x} D^{i+1}\left(\Lambda_{n}^{h, k} f-f\right)(z) d z \\
= & -\int_{x}^{t_{1}} D^{i+1}\left(\Lambda_{n}^{h, k} f-f\right)(z) d z \\
= & \sigma_{i} \int_{x}^{t_{1}} \sigma_{s(i)} D^{i+1}\left(\Lambda_{n}^{h, k} f-f\right)(z) d z .
\end{aligned}
$$

Using (10) we get $\sigma_{i} D^{i}\left(\Lambda_{n}^{h, k} f-f\right) \geq 0$ on $\left[t_{0}, t_{1}\right]$, or $\sigma_{i} D^{i} \Lambda_{n}^{h, k} f \geq \sigma_{i} D^{i} f$ on $\left[t_{0}, t_{1}\right]$; that is, $\Lambda_{n}^{h, k} f \in \sigma_{i} \Delta^{i}\left[t_{0}, t_{1}\right]$ if $f \in \sigma_{i} \Delta^{i}\left[t_{0}, t_{1}\right]$.

It can be shown analogously (by induction with $j$ as induction variable) that $\Lambda_{n}^{h, k} f \in \sigma_{i} \Delta^{i}\left[t_{j}, t_{j+1}\right]$ if $f \in \sigma_{i} \Delta^{i}\left[t_{j}, t_{j+1}\right]$ for any $j=1, \ldots, n-2$. Therefore $\Lambda_{n}^{h, k}\left(\sigma_{i} \Delta^{i}[0,1]\right) \subset \sigma_{i} \Delta^{i}[0,1]$.

After completing induction steps with $i=k-3, k-4, \ldots, h$ we can conclude that $\Lambda_{n}^{h, k}\left(\Delta^{h, k}(\sigma)\right) \subset \Delta^{h, k}(\sigma)$.

It can be easily verified that

(1) $\Lambda_{n}^{h, k} e_{p}=e_{p}$ for all $p=0,1, \ldots, h-1$ (since $\pm e_{p} \in$ $\Delta^{h, k}(\sigma)$ for all $\left.p=0,1, \ldots, h-1\right)$;
(2) if $x \in\left[t_{j}, t_{j+1}\right)$ for some $0 \leq j \leq n-2$, then

$$
D^{k-2}\left(\Lambda_{n}^{h, k} e_{k}-e_{k}\right)(x)=\frac{k !\left(t_{j+1}-x\right)\left(x-t_{j}\right)}{2} .
$$

It follows from (15) that the inequality

$$
0 \leq D^{k-2}\left(\Lambda_{n}^{h, k} e_{k}-e_{k}\right) \leq \frac{k !}{8}(n-1)^{-2},
$$

holds on $[0,1]$.

Suppose (by induction) that for a fixed $i \geq h+1$ there exist $c_{l}>0, l=i+1, \ldots, k-2$, such that for every $x \in[0,1]$

$$
\begin{array}{r}
0 \leq \sigma_{l} D^{l}\left(\Lambda_{n}^{h, k} f-f\right)(x) \leq \mathcal{c}_{l}(n-1)^{-2}, \\
l=i+1, \ldots, k-2 .
\end{array}
$$

It follows from

$$
\begin{aligned}
D^{i}\left(\Lambda_{n}^{h, k} e_{k}-e_{k}\right)(0)= & D^{i} e_{k}(0) \\
& -\delta_{i} \int_{0}^{1} D^{i+1}\left(\Lambda_{n}^{h, k} e_{k}-e_{k}\right)(t) d t
\end{aligned}
$$

that for $x \in[0,1]$

$$
\begin{aligned}
& \left|D^{i}\left(\Lambda_{n}^{h, k} e_{k}-e_{k}\right)(x)\right| \\
& \quad=\left|D^{i}\left(\Lambda_{n}^{h, k} e_{k}-e_{k}\right)(0)+\int_{0}^{x} D^{i+1}\left(\Lambda_{n}^{h, k} e_{k}-e_{k}\right)(t) d t\right| \\
& \quad=2\left|\int_{0}^{1} D^{i+1}\left(\Lambda_{n}^{h, k} e_{k+2}-e_{k+2}\right)(t) d t\right| \\
& \quad \leq 2 \frac{c_{i+1}}{(n-1)^{2}} \frac{1}{(k-i) !} .
\end{aligned}
$$

We have used the fact that if $f \in C[0,1], f \geq 0$, and there exists a constant $a \in \mathbb{R}$ such that $f \leq a$ on $[0,1]$, then for every $p \in \mathbb{N}$

$$
0 \leq \int_{0}^{x} \int_{0}^{t_{p-1}} \cdots \int_{0}^{t_{1}} f\left(t_{1}\right) d t_{1} \cdots d t_{p} \leq a \frac{x^{p}}{p !} .
$$

Then (19) implies with $c_{i}=2\left(c_{i+1} /(k-i) !\right)$

$$
\begin{gathered}
\left\|D^{i}\left(\Lambda_{n}^{h, k} e_{k+2}\right)-D^{i} e_{k+2}\right\|_{B[0,1]} \leq c_{i}(n-1)^{-2} \\
\left\|\Lambda_{n}^{h, k} e_{k}-e_{k}\right\|_{B^{k-2}[0,1]}:=\sum_{i=0}^{k-2} \frac{1}{i !}\left\|D^{i}\left(\Lambda_{n}^{h, k} e_{k}-e_{k}\right)\right\|_{B[0,1]} \\
\leq(n-1)^{-2} \sum_{i=0}^{k-2} \frac{c_{i}}{i !}
\end{gathered}
$$

Direct verification shows that $\Lambda_{n}^{h, k} e_{p}=e_{p}$ for all $p=$ $h, \ldots, k-1$.

Thus, (9) is verified and theorem is proved. 


\section{The Main Result}

Let $X$ be a linear normed space. Recall that linear $n$-width of a set $A \subset X$ in $X$ is defined by [12]

$$
\delta_{n}(A)_{X}:=\inf _{L_{n}} e\left(A, L_{n}\right),
$$

where infimum is taken over all linear continuous operators $L_{n}: X \longrightarrow X$ of finite rank $n$.

Dealing with the problem of approximation of smooth functions by some class of linear operators, we may find that operators of this class have some property which limits the degree of approximation of smooth functions by operators of this class. Let us cite the well-known instances. By definition, every positive linear operator is shape-preserving with respect to the cone of all nonnegative functions $\Delta^{0}$. It was shown by Korovkin [13] that if linear polynomial operator preserves positiveness, the degree of approximation of continuous functions by this operator is low. He proved that the order of approximation by positive linear polynomial operators of degree $n$ cannot be better than $n^{-2}$ in $C[0,1]$ even for the functions $1, x$, and $x^{2}$. Moreover, Videnskiu [14] has shown that the result of [13] does not depend on the properties of the polynomials but rather on the limitation of dimension.

To determine the negative impact of the property of shape-preserving on the order of linear approximation we introduce the following definition based on ideas of Korovkin.

Let $X$ be a linear normed space. Let $V$ be a cone in $X$ and let $A \subset X$ be a set and $A \cap V \neq \varnothing$.

Definition 2. Let one define Korovkin linear relative $n$-width of set $A$ in $X$ with the constraint $V$ by

$$
\delta_{n}(A, V)_{X}:=\inf _{L_{n}(V) \subset V} e\left(A, L_{n}\right),
$$

where infimum is taken over all linear continuous operators $L_{n}: X \longrightarrow X$ of finite rank $n$ satisfying $L_{n}(V) \subset V$.

Note that if $V=X$ then $\delta_{n}(A, V)_{X}=\delta_{n}(A)_{X}$.

If we compare the value of Korovkin linear relative $n$ width $\delta_{n}(A, V)_{X}$ of set $A$ in $X$ with the constraint $V$ to the value of linear $n$-width $\delta_{n}(A)_{X}$ of the set $A$ in $X$ we can evaluate the negative impact of the shape-preserving constraint $L_{n}(V) \subset V$ on the intrinsic error of approximation by means of the shape-preserving linear operators of finite rank $n$ compared to the error of unconstrained linear finiterank approximation on the same set.

This section examines approximation properties of linear finite dimensional operators $L_{n}$ preserving the cone $\Delta^{h, k}(\sigma)$, that is, such that

$$
L_{n}\left(\Delta^{h, k}(\sigma)\right) \subset \Delta^{h, k}(\sigma) .
$$

In this section we will find estimates of Korovkin linear relative $n$-widths for linear operators preserving the cone $\Delta^{h, k}(\sigma)$ in the space $C^{k-2}[0,1]$, that is, estimates of $\delta_{n}\left(A, \Delta^{h, k}(\sigma)\right)_{C^{k-2}[0,1]}$.

First we will prove a preliminary result.
Lemma 3. Let $L_{n}: C^{k-2}[0,1] \longrightarrow B^{k-2}[0,1]$ be a linear operator of finite rank $n, n>k+2$, satisfying (24). Then there exists $c>0$ such that

$$
\sup _{x \in[0,1]}\left|D^{k-2} L_{n} e_{p}(x)-D^{k-2} e_{p}(x)\right| \geq c n^{-2} \quad \forall h \leq p \leq k .
$$

Proof. (1) It is sufficient to show that (25) holds for any linear operator $L_{n}: C^{k-2}[0,1] \longrightarrow B^{k-2}[0,1]$ of finite rank $n$, such that $L_{n}\left(\Delta^{h, k}(\sigma)\right) \subset \sigma_{k-2} \Delta^{k-2}$. Denote $Z=\left\{z_{i}\right\}$, where $z_{i}=$ $i / n, i=0, \ldots, n$. Let $\left\{v_{1}, \ldots, v_{n}\right\}$ be the system of functions generating the linear space $\left\{D^{k-2} L_{n} f: f \in C[0,1]\right\}$; that is, $\operatorname{span}\left\{v_{1}, \ldots, v_{n}\right\}=\left\{D^{k-2} L_{n} f: f \in C[0,1]\right\}$.

Consider the matrix $A=\left(v_{j}\left(z_{i}\right)\right)_{j=1, \ldots, n}^{i=0, \ldots, n}$. Rank of matrix $A$ is not equal to $0, \operatorname{rank} A \neq 0$. Indeed, if $\operatorname{rank} A=0$, then $D^{k-2} L_{n} f\left(z_{i}\right)=\sum_{j=1}^{n} a_{j}(f) v_{i}\left(z_{i}\right)=0, i=0, \ldots, n$, for every $f \in C^{k-2}[0,1]$, which is impossible.

Take a nontrivial vector $\delta=\left(\delta_{0}, \ldots, \delta_{n}\right) \in R^{n+1}$, such that

$$
\sum_{i=0}^{n}\left|\delta_{i}\right|=1, \quad \sum_{i=0}^{n} \delta_{i} v_{j}\left(z_{i}\right)=0, \quad j=1, \ldots, n .
$$

Let $F_{k-2}(Z, \delta)$ denote the set of functions $f \in C^{k-2}[0,1]$, such that $D^{k-2} f\left(z_{i}\right)=\operatorname{sgn} \delta_{i}, i=0, \ldots, n$. Let a function $g \in F(Z, \delta)$ be such that

$$
\sup _{0 \leq j \leq n-1} \sup _{x \in\left(z_{j}, z_{j+1}\right)}\left[z_{j}, x, z_{j+1}\right]\left(D^{k-2} g\right)=\lambda(Z, \delta),
$$

where

$$
\lambda(Z, \delta):=\inf _{f \in F_{k-2}(Z, \delta)} \max _{0 \leq j \leq n-1} \sup _{x \in\left[z_{j}, z_{j+1}\right]}\left[z_{j}, x, z_{j+1}\right]\left(D^{k-2} f\right),
$$

where points $z_{j}, x$, and $z_{j+1}$ are arranged in ascending order.

Denote by $D_{n}$ the set of all vectors $\delta=\left(\delta_{i}\right)_{i=0}^{n}$ with $\delta_{i} \epsilon$ $\{-1,0,1\}$.

It follows from Theorem 2.1 in [15] that

$$
\max _{0 \leq j \leq n-1} \sup _{x \in\left[z_{j}, z_{j+1}\right]}\left[z_{j}, x, z_{j+1}\right]\left(D^{k-2} g\right) \leq \lambda(Z),
$$

where

$$
\lambda(Z):=\sup _{\delta \in D_{n}} \lambda(\delta, Z) \leq \frac{\left(3 n^{2}\right)}{2} .
$$

It follows from $D^{k-2} L_{n} g \in \operatorname{span}\left\{v_{1}, \ldots, v_{n}\right\}$ that $\sum_{i=1}^{n} \delta_{i}$ $D^{k-2} L_{n} g\left(z_{i}\right)=0$. Then

$$
\begin{aligned}
1 & =\sum_{i=0}^{n}\left|\delta_{i}\right|=\sum_{i=0}^{n} \delta_{i} D^{k-2} g\left(z_{i}\right) \\
& =\sum_{i=0}^{n} \delta_{i}\left(D^{k-2} g\left(z_{i}\right)-D^{k-2} L_{n} g\left(z_{i}\right)\right) \\
& \leqslant \sum_{i=0}^{n}\left|\delta_{i}\right|\left|D^{k-2} L_{n} g\left(z_{i}\right)-D^{k-2} g\left(z_{i}\right)\right| \\
& \leqslant\left\|D^{k-2} L_{n} g-D^{k-2} g\right\|_{B[0,1]} .
\end{aligned}
$$


(2) Given $z \in[0,1]$, we have

$$
\begin{aligned}
\mid & D^{k-2} L_{n} g(z)-D^{k-2} g(z) \mid \\
= & \mid D^{k-2} L_{n}\left(g-\frac{e_{k-2}}{(k-2) !} D^{k-2} g(z)\right)(z) \\
& \quad+\frac{1}{(k-2) !} D^{k-2} g(z)\left(D^{k-2} L_{n} e_{k-2}-D^{k-2} e_{k-2}\right)(z) \mid \\
= & \left|D^{k-2} L_{n}\left(g-\frac{e_{k-2}}{(k-2) !} D^{k-2} g(z)\right)(z)\right| \\
& +\frac{1}{(k-2) !}\left|D^{k-2} g(z)\right|\left|\left(D^{k-2} L_{n} e_{k-2}-D^{k-2} e_{k-2}\right)(z)\right| \\
\leq & \left|D^{k-2} L_{n}\left(g-\frac{e_{k-2}}{(k-2) !} D^{k-2} g(z)\right)(z)\right| \\
& +\frac{1}{(k-2) !}\left|\left(D^{k-2} L_{n} e_{k-2}-D^{k-2} e_{k-2}\right)(z)\right|,
\end{aligned}
$$

since $\left\|D^{k-2} g\right\|_{B[0,1]}=1$.

Denote $g_{z}=g-\left(e_{k-2} /(k-2) !\right) D^{k-2} g(z)$. It follows from [16] that there exist functions $\varphi_{z, j} \in \operatorname{span}\left\{e_{h}, \ldots, e_{k}\right\}, j=1,2$, such that

$$
\varphi_{z, j}+(-1)^{j} g_{z} \in \Delta^{h, k}(\sigma), \quad j=1,2,
$$

and such that

(1) $\varphi_{z, j} \in \Delta^{h, k}\left(\sigma^{(r)}\right)$, where $\sigma^{(j)}:=\left(\sigma_{i}^{(j)}\right)_{i \geq 0}$ with $\sigma_{i}^{(j)}=\sigma_{i}$ for $i \neq j$ and $\sigma_{j}^{(j)}=0$;

(2) $D^{k-2} \varphi_{z, j}(z)=0<D^{k-2} \varphi_{z, j}(x)$ for all $x \in[0,1] \backslash\{z\}$;

(3) $D^{k} \varphi_{z, j}=2 \sigma_{k} \cdot \max _{0 \leq j \leq n-1} \sup _{x \in\left[z_{j}, z_{j+1}\right]}\left[z_{j}, x, z_{j+1}\right]$ $\left(D^{k-2} g\right)$.

Let $b_{i, j}, i=h, \ldots, k$, be such that $\varphi_{z, j}=\sum_{i=h}^{k} b_{i, j} e_{i}, j=1,2$. It follows from the proof of Theorem 2 in [16] that there exist positive real numbers $a_{i}, i=h, \ldots, k$, such that

$$
\left|b_{i, j}\right| \leq a_{i}\left|D^{k} \varphi_{z, j}\right|, \quad j=1,2
$$

Then $L_{n}\left(\varphi_{z, j}+(-1)^{j} g_{z}\right) \in \sigma_{k-2} \Delta^{k}, j=1,2$, and, consequently, we have

$$
\begin{aligned}
& \left|D^{k-2} L_{n}\left(g-\frac{e_{k-2}}{(k-2) !} D^{k-2} g(z)\right)(z)\right| \\
& \quad \leq \max \left\{\left|D^{k-2} L_{n} \varphi_{z, 1}(z)\right|,\left|D^{k-2} L_{n} \varphi_{z, 2}(z)\right|\right\} .
\end{aligned}
$$

It follows from $D^{k-2} \varphi_{z, j}(z)=0$ that

$$
\begin{aligned}
\mid D^{k-2} & L_{n} \varphi_{z, j}(z) \mid \\
& =\left|D^{k-2} L_{n} \varphi_{z, j}(z)-D^{k-2} \varphi_{z, j}(z)\right| \\
\leq & \left\|D^{k-2} L_{n} \varphi_{z, j}-D^{k-2} \varphi_{z, j}\right\|_{B[0,1]} \\
\leq & \sum_{i=h}^{k}\left|b_{i, j}\right|\left\|D^{k-2} L_{n} e_{i}-D^{k-2} e_{i}\right\|_{B[0,1]} \\
\leq & \left|D^{k} \varphi_{z, j}\right| \sum_{i=h}^{k}\left|a_{i}\right|\left\|D^{k-2} L_{n} e_{i}-D^{k-2} e_{i}\right\|_{B[0,1]} \\
\leq & 2 \max _{0 \leq j \leq n-1} \sup _{x \in\left[z_{j}, z_{j+1}\right]}\left[z_{j}, x, z_{j+1}\right]\left(D^{k-2} g\right) \\
& \times \sum_{i=h}^{k}\left|a_{i}\right|\left\|D^{k-2} L_{n} e_{i}-D^{k-2} e_{i}\right\|_{B[0,1]},
\end{aligned}
$$

where $a_{i}, i=h, \ldots, k$, do not depend on $n$.

It follows from (36) and (29) that

$$
\begin{aligned}
& \left|D^{k-2} L_{n} \varphi_{z, j}(z)\right| \\
& \quad \leq 2 \lambda(Z) \sum_{i=h}^{k}\left|a_{i}\right|\left\|D^{k-2} L_{n} e_{i}-D^{k-2} e_{i}\right\|_{B[0,1]} .
\end{aligned}
$$

It follows from (31), (32), (35), (36), and (37) that

$$
\frac{1}{2 \lambda(Z)} \leq \sum_{i=h}^{k}\left|a_{i}\right|\left\|D^{k-2} L_{n} e_{i}-D^{k-2} e_{i}\right\|_{B[0,1]} .
$$

Thus, as it follows from (30) there exists a constant $C>0$ such that

$$
C n^{-2} \leq \sum_{i=h}^{k}\left\|D^{k-2} L_{n} e_{i}-D^{k-2} e_{i}\right\|_{B[0,1]} .
$$

Theorem 4. Let $n \geq k+2$. If $\sigma_{k} \sigma_{k-2}=1$ then

(1) there exist $c_{1}, c_{2}>0$ not depending on $n$ such that

$$
c_{1} n^{-2}<\delta\left(P_{k}, \Delta^{h, k}(\sigma)\right)_{C^{k-2}[0,1]}<c_{2} n^{-2}
$$

(2) for all $0 \leq m \leq k-1$

$$
\delta_{n}\left(P_{m}, \Delta^{h, k}(\sigma)\right)_{C^{k}[0,1]}=0 .
$$

If $\sigma_{k} \sigma_{k-2} \neq 1$ then

$$
\delta\left(P_{k}, \Delta^{h, k}(\sigma)\right)_{C^{k-2}[0,1]}=0 .
$$


Proof. To prove (40) it is sufficient to show that there exist $c_{1}, c_{2}>0$ not depending on $n$ such that

$$
c_{1} n^{-2}<\inf _{L_{n}\left(\Delta^{h, k}(\sigma)\right) \subset \Delta^{h, k}(\sigma)} \sup _{f \in P_{k}}\left\|f-L_{n} f\right\|_{B^{k-2}[0,1]}<c_{2} n^{-2}
$$

where infimum is taken over all linear continuous operators $L_{n}$ of $C^{k-2}[0,1]$ into $B^{k-2}[0,1]$ of finite rank $n$ satisfying $L_{n}\left(\Delta^{h, k}(\sigma)\right) \subset \Delta^{h, k}(\sigma)$. Without loss of generality we will assume $\sigma_{k}=\sigma_{k-2}=1$. Then the upper estimate in (40) follows from Theorem 1 . The lower estimate follows from Lemma 3.

As it was shown in the proof of Theorem 1 there exists a continuous linear operator $\Lambda_{n}^{h, k}: C^{k-2}[0,1] \longrightarrow C^{k-2}[0,1]$ of finite rank $n$, such that $\Lambda_{n}^{h, k}\left(\Delta^{h, k}(\sigma)\right) \subset \Delta^{h, k}(\sigma)$, and for all $0 \leq i \leq k-2$

$$
\sup _{f \in P_{m}}\left\|D^{i}\left(\Lambda_{n}^{h, k} f\right)-D^{i} f\right\|_{B[0,1]}=0, \quad 0 \leq m \leq k-1 .
$$

It follows from (44) that

$$
\begin{array}{r}
\inf _{L_{n}\left(\Delta^{h, k}(\sigma)\right) \subset \Delta^{h, k}(\sigma)} \sup _{f \in P_{m}}\left\|f-L_{n} f\right\|_{B^{k-2}[0,1]}=0, \\
0 \leq m \leq k-1,
\end{array}
$$

where infimum is taken over all linear continuous operators $L_{n}$ of $C^{k-2}[0,1]$ into $B^{k-2}[0,1]$ of finite rank $n$ satisfying $L_{n}\left(\Delta^{h, k}(\sigma)\right) \subset \Delta^{h, k}(\sigma)$, and consequently (41) holds.

Finally, (42) follows from [16], where it is shown (remark after Proposition 1) that there exists a linear operator that maps the cone of positive and concave functions onto the same cone and holds the space $P_{3}$.

$$
\text { Denote } B^{(k)}:=\left\{f \in B^{k}[0,1]:\|f\|_{B^{k}[0,1]} \leq 1\right\} .
$$

Theorem 5. Let $n \geq k+2$ and $\sigma_{k} \sigma_{k-2}=1$. Then there exist $c_{1}, c_{2}>0$ not depending on $n$ such that

$$
c_{1} n^{-2}<\delta_{n}\left(B^{(k)}, \Delta^{h, k}(\sigma)\right)_{C^{k-2}[0,1]}<c_{2} n^{-2}
$$

Proof. Note that $P_{k} \subset B^{(k)}$. Then the lower estimate in (46) follows from (40).

On the other hand, if $f \in B^{(k)}$ and $x, t \in[0,1]$ then

$$
f(t)=\sum_{r=0}^{k-1} \frac{D^{r} f(x)}{r !} x^{r}+\frac{1}{k !} \int_{0}^{1}(x-t)_{+}^{k} D^{k} f(t) d t
$$

and $\left\|D^{k} f\right\|_{B[0,1]} \leq 1$. The properties of linear operator $\Lambda_{n}^{h, k}$ defined in (8) imply that there exists $c_{2}>0$ such that

$$
\left\|f-\Lambda_{n}^{h, k} f\right\|_{B^{k-2}[0,1]} \leq \frac{c_{2}}{(n-1)^{2}} .
$$

\section{Conclusion}

Estimation of linear relative $n$-widths is of interest in the theory of shape-preserving approximation as, knowing the value of relative linear $n$-width, we can judge how good or bad (in terms of optimality) this or that finite dimensional method preserving the shape in the sense $V$ is.

The paper shows that if a linear operator with finite rank $n$ preserves the shape in the sense of cone $\Delta^{h, k}(\sigma)$, the degree of simultaneous approximation of derivatives of order $0 \leq i \leq k-2$ of continuous functions by derivatives of this operator cannot be better than $n^{-2}$ on both the set $P_{k}$ and the ball $B^{(k)}$. Results show that the shape-preserving property of operators is negative in the sense that the error of approximation by means of such operators does not decrease with the increase of smoothness of approximated functions. In other words, there is saturation effect for linear finite-rank operators preserving the shape in the sense of cone $\Delta^{h, k}(\sigma)$. It is worth noting that nonlinear approximation preserving $k$-monotonicity does not have this shortcoming [17].

\section{Conflict of Interests}

The author declares that there is no conflict of interests regarding the publication of this paper.

\section{Acknowledgment}

This work is supported by RFBR (Grants 14-01-00140 and 1301-00238).

\section{References}

[1] S. G. Gal, Shape-Preserving Approximation by Real and Complex Polynomials, Springer, 2008.

[2] V. N. Konovalov, "Estimates of diameters of Kolmogorov type for classes of differentiable periodic functions," Mathematical Notes of the Academy of Sciences of the USSR, vol. 35, no. 3, pp. 369-380, 1984.

[3] V. N. Konovalov and D. Leviatan, "Shape preserving widths of Sobolev-type classes of $s$-monotone functions on a finite interval," Israel Journal of Mathematics, vol. 133, pp. 239-268, 2003.

[4] J. Gilewicz, V. N. Konovalov, and D. Leviatan, "Widths and shape-preserving widths of Sobolev-type classes of $s$-monotone functions," Journal of Approximation Theory, vol. 140, no. 2, pp. 101-126, 2006.

[5] V. N. Konovalov and D. Leviatan, "Shape-preserving widths of weighted Sobolev-type classes of positive, monotone, and convex functions on a finite interval," Constructive Approximation, vol. 19, no. 1, pp. 23-58, 2003.

[6] Yu. N. Subbotin and S. A. Telyakovskiü, "Exact values of relative widths of classes of differentiable functions," Mathematical Notes, vol. 65, no. 6, pp. 871-879, 1999.

[7] Y. Subbotin and S. Telyakovskii, "Splines and relative widths of classes of differentiable functions," Proceedings of the Institute of Mathematics and Mechanics, vol. 7, pp. S225-S234, 2001.

[8] Yu. N. Subbotin and S. A. Telyakovskiı̌, "Relative widths of classes of differentiable functions in the $L^{2}$ metric," Russian Mathematical Surveys, vol. 56, no. 4, pp. 159-160, 2001. 
[9] Yu. N. Subbotin and S. A. Telyakovskiü, "On the relative widths of classes of differentiable functions," Proceedings of the Steklov Institute of Mathematics, vol. 248, no. 1, pp. 250-261, 2005.

[10] Yu. N. Subbotin and S. A. Telyakovski1̌, "On the equality of Kolmogorov and relative widths of classes of differentiable functions," Mathematical Notes, vol. 86, no. 3, pp. 456-465, 2009.

[11] Yu. N. Subbotin and S. A. Telyakovskiĭ, "Sharpening of estimates for the relative widths of classes of differentiable functions," Proceedings of the Steklov Institute of Mathematics, vol. 269, no. 1, pp. 242-253, 2010.

[12] V. M. Tikhomirov, Some Problems in Approximation Theory, Izd-vo Moskovskogo Universiteta, 1976.

[13] P. P. Korovkin, "On the order of the approximation of functions by linear positive operators," Doklady Akademii Nauk SSSR, vol. 114, pp. 1158-1161, 1957.

[14] V. S. Videnskil̆, "On an exact inequality for linear positive operators of finite rank," Doklady Akademii Nauk Tadzhikskǒ SSR, vol. 24, no. 12, pp. 715-717, 1981.

[15] S. P. Sidorov and V. Balash, "Estimates of divided differences of real-valued functions defined with a noise," International Journal of Pure and Applied Mathematics, vol. 76, no. 1, pp. 95106, 2012.

[16] F. J. Muñoz-Delgado, V. Ramírez-González, and D. CárdenasMorales, "Qualitative Korovkin-type results on conservative approximation," Journal of Approximation Theory, vol. 94, no. 1, pp. 144-159, 1998.

[17] K. Kopotun and A. Shadrin, "On k-monotone approximation by free knot splines," SIAM Journal on Mathematical Analysis, vol. 34, no. 4, pp. 901-924, 2003. 


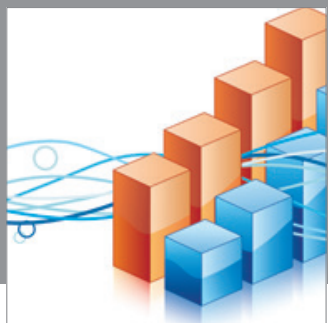

Advances in

Operations Research

mansans

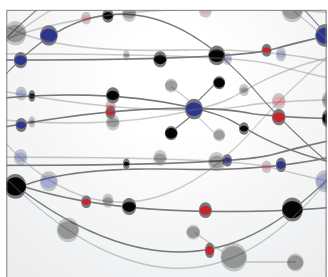

The Scientific World Journal
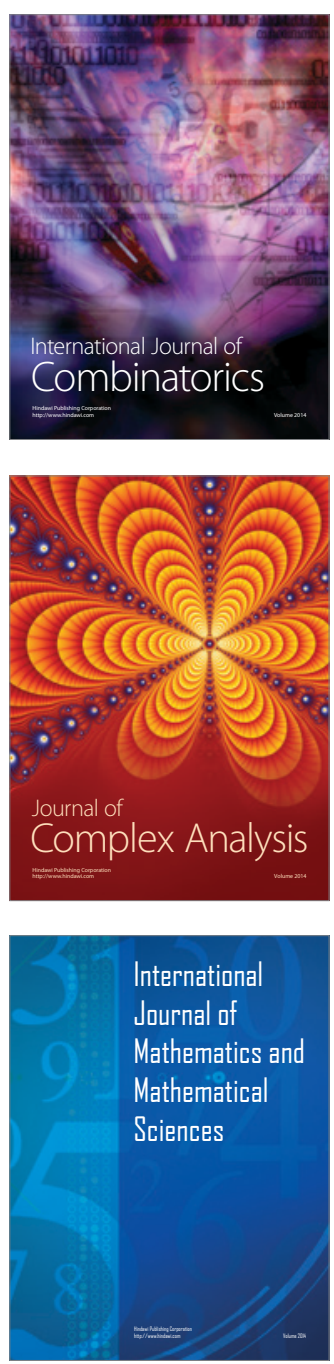
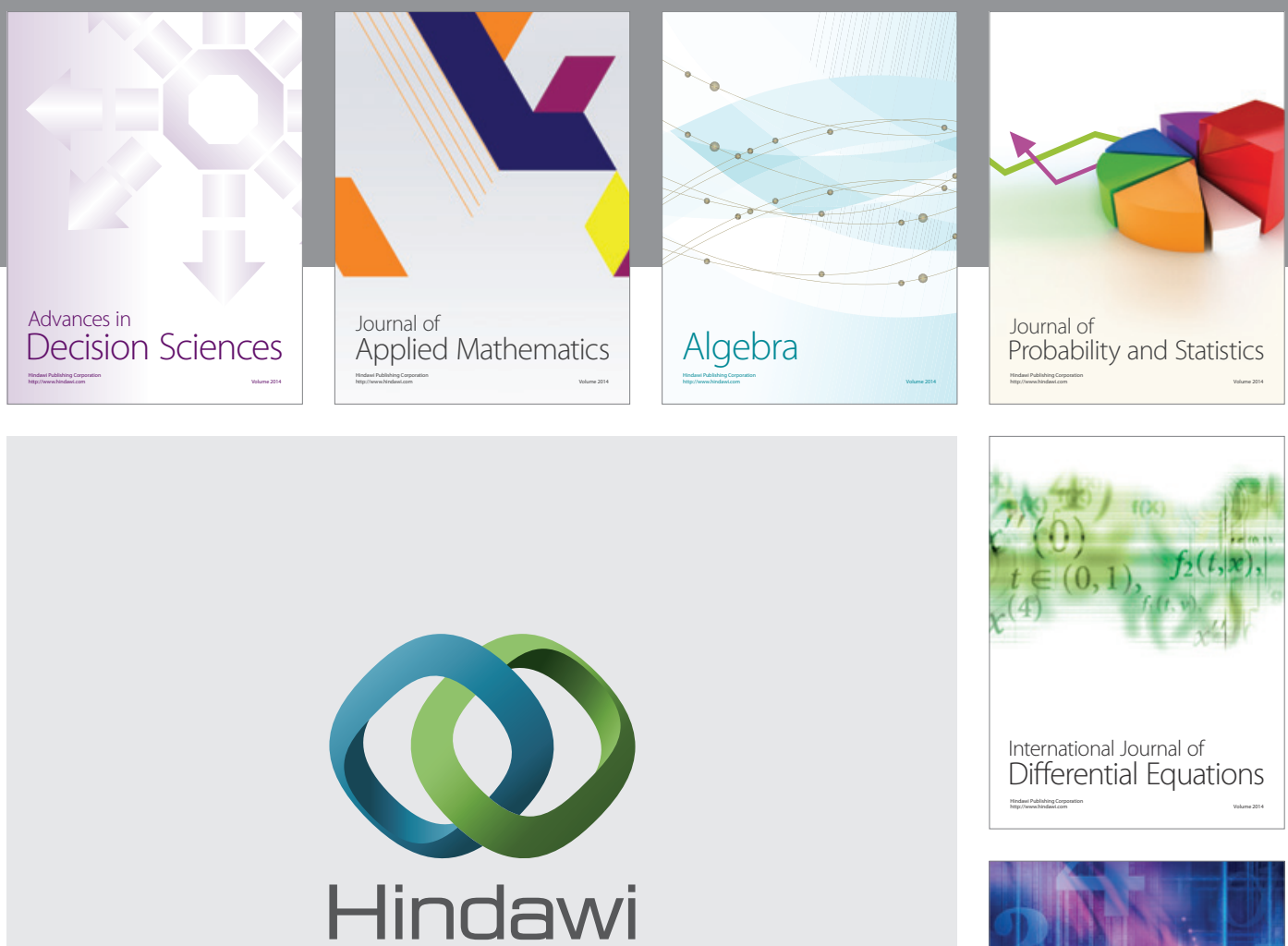

Submit your manuscripts at http://www.hindawi.com
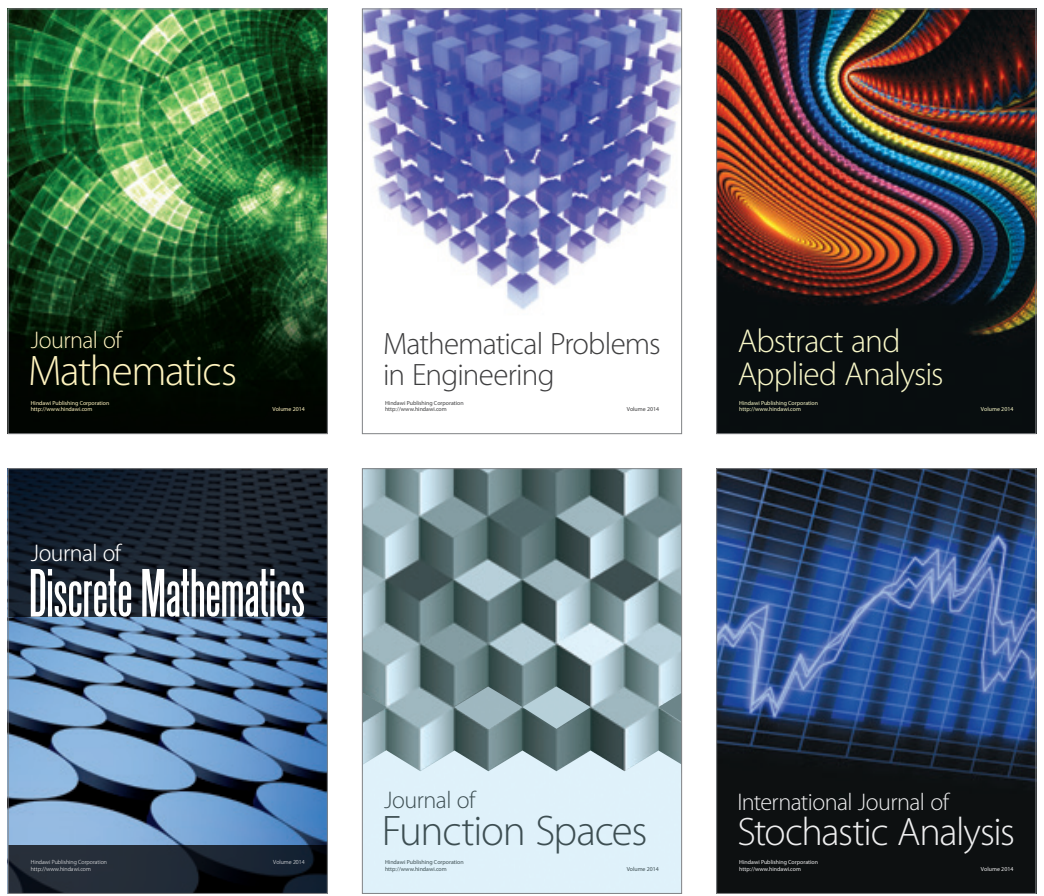

Journal of

Function Spaces

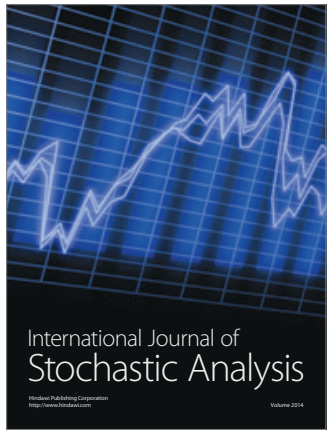

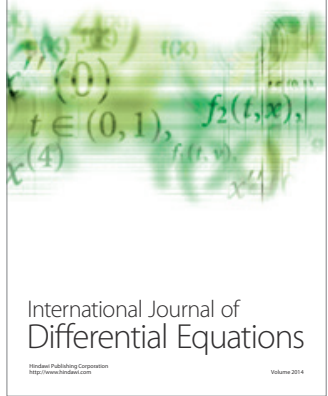
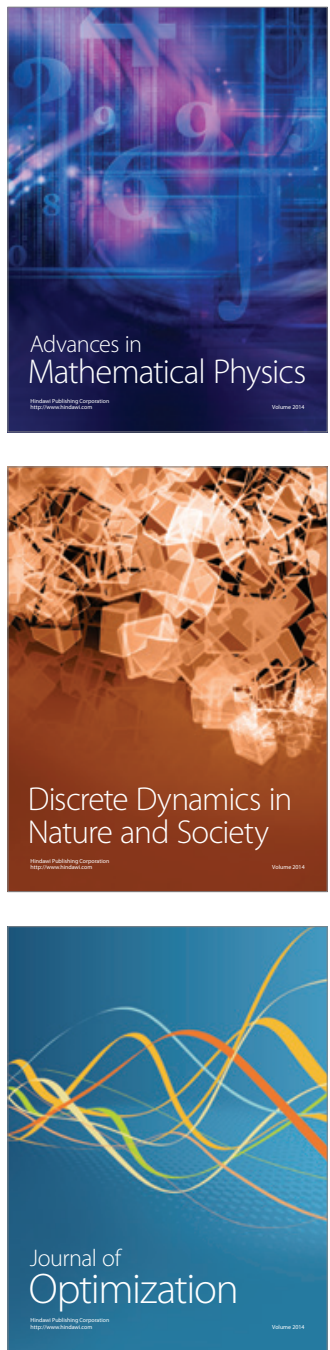